\title{
ON DIFFERENTIAL EQUATIONS OF MODE 2
}

\author{
JOHANNES C. C. NITSCHE ${ }^{1}$
}

It is a well-known fact, documented by striking examples, that the solutions of sufficiently nonlinear elliptic differential equations behave quite differently from the solutions of linear elliptic differential equations. Directing one's attention to the study of certain properties of the solutions of certain classes of differential equations, one actually can define measures of nonlinearity, conditions which, when fulfilled, guarantee the validity of the properties under consideration. This has been done by a number of authors in various ways and in regard to different properties of interest-solvability of Dirichlet's problem, possibility of isolated singularities, existence of nonlinear entire solutions, validity of certain a priori estimates, etc.-see for instance S. Bernstein [1], L. Bers [3], R. Finn [4], [5], [6], [7], [8], D. Gilbarg [9], H. Jenkins [10], [11], [12], H. Jenkins and J. Serrin [13], J. Leray [14], Johannes and Joachim Nitsche [16], [17], J. Serrin [18], [19]. Of course, in most cases these measures only lead to sufficient conditions.

The first such discussion was carried out by S. Bernstein in 1912 (see [1, especially pp. 455-469]) for quasi-linear elliptic differential equations

$$
L[z] \equiv a(x, y ; p, q) r+2 b(x, y ; p, q) s+c(x, y ; p, q) t=0,
$$

where $a c-b^{2}>0, a>0$, and in greater generality by J. Leray (see [14, especially pp. 281-283]). Let us say that a function $F(\xi, \eta)$ has a definite growth for $\xi$ and $\eta$ tending to infinity, and that this growth is of order $\alpha$ (in signs $\left.F \sim\left(\xi^{2}+\eta^{2}\right)^{\alpha / 2}\right)$, if there are two constants $m, \rho_{0}\left(1 \leqq m<\infty, \rho_{0}>0\right)$, and an exponent $\alpha$ such that the inequalities

$$
\frac{1}{m}\left(\xi^{2}+\eta^{2}\right)^{\alpha / 2} \leqq F(\xi, \eta) \leqq m\left(\xi^{2}+\eta^{2}\right)^{\alpha / 2}
$$

hold for $\xi^{2}+\eta^{2} \geqq \rho_{0}^{2}$. Under certain regularity conditions and assuming that the two forms

$$
\begin{aligned}
& F_{1}=F_{1}(x, y ; p, q) \equiv a p^{2}+2 b p q+c q^{2}, \\
& F_{2}=F_{2}(x, y ; p, q) \equiv(a+c)\left(p^{2}+q^{2}\right)
\end{aligned}
$$

Received by the editors July 10, 1964.

1 The preparation of this paper has been supported in part by Air Force Grant AF-AFOSR-883-65 and in part by Contract $710(54)$ between the University of Minnesota and the Office of Naval Research. 
have, uniformly for all $(x, y)$ in the domain under consideration, a definite growth as $p$ and $q$ tend to infinity, of orders $\mu_{1}$ and $\mu_{2}$, respectively, S. Bernstein calls the difference $\mu=\mu_{2}-\mu_{1}$ the mode ("genre") of the differential equation (1). From the relation $F_{2}-F_{1}=a q^{2}$ $-2 b p q+c p^{2}$ it follows that $F_{2}>F_{1}$ for $p^{2}+q^{2} \neq 0$ and that $\mu_{2} \geqq \mu_{1}$, i.e. $\mu \geqq 0$. For the minimal surface equation we obtain $F_{1}=p^{2}+q^{2}$, $F_{2}=\left(p^{2}+q^{2}\right)\left(2+p^{2}+q^{2}\right)$, so that its mode is two. Linear and uniformly elliptic differential equations are of mode zero.

The investigations of $\mathrm{S}$. Bernstein concerning the solvability of Dirichlet's problem reveal that differential equations whose mode does not exceed the value one behave similar to linear differential equations, while differential equations of a mode exceeding the value one have different features. A particularly interesting class is that of the equations of mode two, the minimal surface equation being a representative of this class.

In 1954 and the following years R. Finn [5], [6], [7], [8] introduced and investigated a class of quasi-linear differential equations which he, on account of their relationship to the minimal surface equation, called equations of minimal surface type: An equation (1), under the normalization $a c-b^{2}=1$ and under varying further conditions, is called of minimal surface type if there is a constant $K(1 \leqq K<\infty)$ such that the inequality

(3) $\quad F_{3}=F_{3}(x, y ; p, q) \equiv a\left(1+p^{2}\right)+2 b p q+c\left(1+q^{2}\right) \leqq 2 K W$

holds for all $x, y$ in the domain under consideration and for all $p, q$. Here $W=\left(1+p^{2}+q^{2}\right)^{1 / 2}$. Note that $F_{2}=\left(p^{2}+q^{2}\right)\left(F_{3}-F_{1}\right)$.

Condition (3) has an interesting geometrical interpretation. It never seems to have been observed, however, that any equation of minimal surface type is an equation of mode 2.

This can be proved in the following way. Setting $\omega=\left(p^{2}+q^{2}\right)^{1 / 2}$ we have

$$
F_{1} F_{2}=\omega^{2}\left[(a p+b q)^{2}+(b p+c q)^{2}+\omega^{2}\right] \geqq \omega^{4}
$$

and, for $\omega^{2} \geqq 1 / 3$,

$$
\omega^{2} F_{1}+F_{2}=\omega^{2} F_{3} \leqq 2 K \omega^{2} W \leqq 4 K \omega^{3} .
$$

From these relations the inequalities $F_{2} \geqq \omega^{4} / F_{1}$ and $\omega^{2} F_{1}+\omega^{4} / F_{1}$ $\leqq 4 K \omega^{3}$ or $F_{1} / \omega+\omega / F_{1} \leqq 4 K$, i.e.

$$
\frac{1}{m} \omega \leqq F_{1} \leqq m \omega, \quad m=2 K+\left(4 K^{2}-1\right)^{1 / 2},
$$

can be concluded. Thus $F_{1}$ has a definite growth of order $\mu_{1}=1$ for 
$\omega \rightarrow \infty$. Now it follows from (4) and (5) that

$$
\frac{1}{m} \omega^{3} \leqq \frac{\omega^{4}}{F_{1}} \leqq F_{2} \leqq 4 K \omega^{3}-\omega^{2} F_{1} \leqq\left(4 K-\frac{1}{m}\right) \omega^{3}=m \omega^{8},
$$

i.e. that $F_{2}$ has a definite growth of order $\mu_{2}=3$ for $\omega \rightarrow \infty$. The mode of the differential equation is therefore $\mu=\mu_{2}-\mu_{1}=2$.

The converse is not true, i.e. differential equations of mode two constitute a larger class than differential equations of minimal surface type, as may be illustrated by the example of the differential equation (1) with coefficients

$$
\begin{aligned}
& a=\frac{1}{d}\left[1+W^{2(\alpha-1)}\left(-q+\beta \frac{p}{W}\right)^{2}\right], \\
& b=\frac{1}{d} W^{2(\alpha-1)}\left(-q+\beta \frac{p}{W}\right)\left(p+\beta \frac{q}{W}\right), \\
& c=\frac{1}{d}\left[1+W^{2(\alpha-1)}\left(p+\beta \frac{q}{W}\right)^{2}\right]
\end{aligned}
$$

where $d=\left[1+\omega^{2} W^{2(\alpha-1)}\left(1+\beta^{2} W^{-2}\right)\right]^{1 / 2}$, so that $a c-b^{2}=1$, and $\alpha>1$. Here we find $F_{1} \sim \omega^{\alpha}, F_{2} \sim \omega^{\alpha+2}, F_{3} \sim \omega^{\alpha}$.

In a famous paper [2], L. Bers proved in 1951 that an isolated singularity of a solution of the minimal surface equation, interior to the domain of definition, ipso facto must be removable. In particular, a solution of the minimal surface equation remains bounded in a neighborhood of such a singularity - a fact which may be interpreted as an extended maximum principle:

Let $z(x, y)$ be a twice continuously differentiable solution of the minimal surface equation (i.e., equation (1) with coefficients $\left.a=\left(1+q^{2}\right) / W, b=-p q / W, c=\left(1+p^{2}\right) / W\right)$ in the punctured disc $P_{0}=\left\{x, y ; 0<x^{2}+y^{2} \leqq 1\right\}$. Let $m=\lim \inf z(x, y)$ and $M=\lim \sup z(x, y)$ for any approach to the circle $x^{2}+y^{2}=1$. Then the inequalities $m \leqq z(x, y) \leqq M$ hold in all of $P_{0}$.

Later the extended maximum principle was proved for more general differential equations by R. Finn [4] using certain level line arguments. In [15], [16] another proof was given for differential equations (1) arising from a variational problem $\delta \iint F\left(p^{2}+q^{2}\right) d x d y=0$. This proof, which also handles singularities on the boundary of the domain of definition, is based on the construction of a certain auxiliary function $\phi$, depending on the variable $r=\left(x^{2}+y^{2}\right)^{1 / 2}$ alone. $\phi$ is a super-solution for the differential equation with certain growth properties. Recently the idea of proving the extended maximum principle 
with the help of such an auxiliary function has been taken up again by R. Finn [8] and H. Jenkins [12]. The function $\phi=\phi(r ; a)$ used by these authors is a twice continuously differentiable solution or supersolution of (1) in an annulus $0<a<r<1$, continuous in the annulus $a<r \leqq 1$, and satisfies the conditions $\phi(1 ; a)=0, \lim _{r \rightarrow a} \phi^{\prime}(r ; a)=-\infty$, and $\lim _{a \rightarrow 0} \phi(r ; a)=0$ for every fixed $r$ in $0<r<1$. With the help of this function the proofs become particularly simple.

We remark that it is immaterial for the proof of the extended maximum principle whether the value $\phi(a ; a)=\lim _{r \rightarrow a} \phi(r ; a)$ is finite or infinite. Of course, it is in the case $\phi(a ; a)<\infty$ that the condition $\lim _{r \rightarrow a} \phi^{\prime}(r ; a)=-\infty$ has relevance.

It seems to have gone unnoticed, incidentally, that the construction of just such a function, i.e. a solution $\phi$ whose gradient tends to infinity upon approach to a certain part of the boundary of the domain of definition, as well as the idea of its employment for the proof of a general maximum principle and for the discussion of the solvability of Dirichlet's problem, already play a basic rôle in Bernstein's investigations of nonlinear differential equations (see [1, esp. pp. 465-469]).

For the minimal surface equation the catenoid has the desired properties and was recently used by R. Finn [8] for a simple proof of the extended maximum principle. The existence of the auxiliary function $\phi$ for more general differential equations depends on the realization of certain conditions. Its construction is possible if the differential equation (1), which we again assume subject to the normalization $a c-b^{2}=1$, is of minimal surface type or, more generally, if the function

$$
E(\omega)=\max _{v^{2}+q^{2}=\omega^{2}}\left\{\sup _{(x, y)} \frac{F_{3}(x, y ; p, q)}{\left(1+p^{2}+q^{2}\right)^{1 / 2}}\right\}
$$

grows so slowly that $\int^{\infty} E^{2}(\omega) \omega^{-3} d \omega$ converges (see H. Jenkins [12]).

As our example (7), for $\alpha \geqq 2$, shows, equations of mode two do not, in general, satisfy this condition. Nevertheless, the extended maximum principle is true also for equations of mode two, in fact, for equations of positive mode and, more generally, for equations satisfying condition (13), as we shall now prove.

For a function $u=u(r)$, depending only on $r=\left(x^{2}+y^{2}\right)^{1 / 2}$, we obtain

$$
L[u]=\frac{F_{1}}{u^{\prime 2}}\left\{u^{\prime \prime}+\left(\frac{F_{2}}{F_{1}}-1\right) \frac{u^{\prime}}{r}\right\}
$$

and, setting $v=u^{\prime 2}$, 


$$
L[u]=\frac{F_{1}}{2 u^{\prime 3}}\left\{v^{\prime}+\frac{2 v}{r}\left(\frac{F_{2}}{F_{1}}-1\right)\right\} .
$$

If we now want a super-solution $u(r)$ with negative $r$-derivative, we must have $L[u] \leqq 0$, i.e.

Let us set

$$
v^{\prime}+\frac{2 v}{r}\left(\frac{F_{2}}{F_{1}}-1\right) \geqq 0
$$

$$
Q(\omega)=\min _{p^{2}+q^{2}=\omega^{2}}\left\{\inf _{(x, y)}\left(\frac{F_{2}(x, y ; p, q)}{F_{1}(x, y ; p, q)}-1\right)\right\}
$$

Then the equation

$$
\frac{d v}{v Q(\sqrt{ } v)}=-2 \frac{d r}{r}
$$

or, in integrated form,

$$
\text { (10) } \quad r=\exp \left\{-\frac{1}{2} \int_{v(1)}^{v(r)} \frac{d v}{v Q(\sqrt{ } v)}\right\}=\exp \left\{-\int_{\left|u^{\prime}(1)\right|}^{\left|u^{\prime}(r)\right|} \frac{d \omega}{\omega Q(\omega)}\right\}
$$

will lead it a super-solution $u(r)$ with $u^{\prime}(r)<0$. For differential equa tions of mode 2 , for which $Q(\omega) \sim \omega^{2}$, the improper integral

$$
\chi(\alpha)=\int_{\alpha}^{\infty} \frac{d \omega}{\omega Q(\omega)}
$$

is finite for $\alpha>0$.

Denoting by $\lambda_{\max }=\lambda_{\max }(x, y ; p, q)$ the larger eigenvalue of the quadratic form $F_{1}$, we find

$$
\frac{F_{2}}{F_{1}}-1 \geqq \frac{(a+c)\left(p^{2}+q^{2}\right)}{\lambda_{\max }\left(p^{2}+q^{2}\right)}-1=\left(\frac{2}{a+c+\left((a+c)^{2}-4\right)^{1 / 2}}\right)^{2} .
$$

If we require that the coefficients $a(x, y ; p, q), b(x, y ; p, q)$ and $c(x, y ; p, q)$ be continuous functions of all variables in $x^{2}+y^{2} \leqq 1$, $p^{2}+q^{2}<\infty$ the existence of a function $\nu(\omega)$, continuous and positive for all $\omega$ in $0 \leqq \omega<\infty$ follows, for which $Q(\omega) \geqq \nu(\omega)$. Thus the function $\chi(\alpha)$ is monotonously decreasing in $0<\alpha<\infty$ and $\lim _{\alpha \rightarrow 0} \chi(\alpha)=\infty$. Let $\psi(\beta)$ be the inverse function of $\chi(\alpha)$. Also $\psi(\beta)$ is monotonously decreasing, and $\lim _{\beta \rightarrow \infty} \psi(\beta)=0$.

For $0<a<1$ denote by $u=\phi(r ; a)$ the solution arising from (10) which is defined in the annulus $a<r \leqq 1$ and satisfies the conditions $\phi(1 ; a)=0, \phi^{\prime}(1 ; a)=-\alpha=-\psi(\log 1 / a), \phi^{\prime}(r, a)<0$ in $a<r<1$, 
$\lim _{r \rightarrow a} \phi^{\prime}(r, a)=-\infty$. We find

$$
\phi(r ; a)=\int_{r}^{1} \psi\left(\log \frac{r^{\prime}}{a}\right) d r^{\prime} .
$$

Considering the properties of the function $\psi$ we see that $\lim _{a \rightarrow 0} \phi(r ; a)$ $=0$ for every fixed $r$ in $0<r \leqq 1$. This completes the proof.

It is interesting to note that the condition

$$
\int^{\infty} \frac{d \omega}{\omega Q(\omega)}<\infty
$$

is identical with the condition $F_{p}^{2}+F_{a}^{2} \leqq M<\infty$ for the special class of differential equations (1), which arise as Euler equations from a variational problem $\delta \iint F\left(p^{2}+q^{2}\right) d x d y=0$. For these equations condition (13) is known to be necessary and sufficient for the extended maximum principle to hold, see [16].

For small $\beta$ (and $\mu>0$ ) the function $\psi(\beta)$ behaves like $c \beta^{-1 / \mu}$. From (12) it then follows that $\phi(a ; a)=\infty$ for $0<\mu \leqq 1$ and $\phi(a ; a)<\infty$ for $\mu>1$ (in fact for equations (1) with $\int^{\infty} d \omega / Q(\omega)<\infty$ ).

REMARK 1 . The preceding considerations carry over immediately to the $n$-dimensional case.

REMARK 2. One could generalize Bernstein's concept of the mode of an equation (1) by considering the behavior of the integral

$$
\int^{\infty} \frac{d \omega}{\omega^{e} Q(\omega)}
$$

rather than the growth of the function $Q(\omega)$.

\section{BIBLIOGRAPHY}

1. S. Bernstein, Sur les équations du calcul des variations, Ann. Sci. École Norm. Sup. (3) 29 (1912), 431-485.

2. L. Bers, Isolated singularities of minimal surfaces, Ann. of Math. 53 (1951), 364-386.

3. - Non-linear elliptic equations without non-linear entire solutions, J. Rational Mech. Anal. 3 (1954), 767-787.

4. R. Finn, Isolated singularities of solutions of non-linear partial differential equations, Trans. Amer. Math. Soc. 75 (1953), 385-404. 416.

5. - On equations of minimal surface type, Ann. of Math. 60 (1954), 397-

6. - On a problem of type, with application to elliptic partial differential equations, J. Rational Mech. Anal. 3 (1954), 789-799.

7. - New estimates for equations of minimal surface type, Arch. Rational Mech. Anal. 14 (1963), 337-375.

8. - Remarks relevant to minimal surfaces, Tech. Rep. No. 120, Appl. Math. and Stat. Lab., Stanford Univ., Stanford, Calif., 1963. 
9. D. Gilbarg, Some local properties of elliptic equations, pp. 127-141, Partial differential equations, Proc. Sympos. Pure Math., Vol. 4, Amer. Math. Soc., Providence, R. I., 1961.

10. H. Jenkins, On two-dimensional variational problems in parametric form, Arch. Rational Mech. Anal. 8 (1961), 181-206.

11. - On quasi-linear elliptic equations which arise from variational problems, J. Math. Mech. 10 (1961), 705-728.

12. - Super solutions for quasi-linear elliptic equations, Arch. Rational Mech. Anal. 16 (1964), 402-410.

13. H. Jenkins and J. Serrin, Variational problems of minimal surface type. I, Arch. Rational Mech. Anal. 12 (1963), 185-212.

14. J. Leray, Discussions d'un probleme de Dirichlet, J. Math. Pures Appl. 18 (1939), 249-284.

15. J. C. C. Nitsche, $Z u$ einem Satze von L. Bers über die Lösungen der Minimalflächengleichung, Arch. Math. 9 (1958), 427-429.

16. Johannes and Joachim Nitsche, Über reguläre Variationsprobleme, Rend. Circ. Mat. Palermo (2) 8 (1959), 346-353.

17. - Ein Kriterium für die Existenz nichtlinearer ganzer Lösungen elliptischer Differentialgleichungen, Arch. Math. 10 (1959), 294-297.

18. J. Serrin, Dirichlet's principle in the calculus of variations, pp. 17-22, Partial differential equations, Proc. Sympos. Pure Math., Vol. 4, Amer. Math. Soc., Providence, R. I., 1961.

19. - Local behavior of solutions of quasi-linear equations, Acta Math. 111 (1964), 247-302.

UNIVERSITY OF MINNESOTA 